\begin{abstract}
The use of multiple antennas in two-hop amplify-and-forward relay selection is analysed, where the source, relay and destination are each equipped with multiple receive but single transmit antennas. Since relay switching is based upon fed back information which is delay-limited, channel power prediction is employed to mitigate against the effect of outdated channel state information being used to make switching decisions. During transmission, a source selects a best relay on the basis of predicted SNR (signal to noise ratio) over all available links. A chosen relay then employs maximal ratio combining at its receiver, and applies a variable gain to the received signal before forwarding to the destination. Closed form outage probability and bit error rate solutions are found for arbitrary numbers of relays and receive antennas, and used to explore trade offs between number of relays and number of antennas compared to single antenna alternatives. To assess predictor performance in combatting switching delay, comparison is made to non-predictive systems.
\end{abstract}




\title{
Channel Prediction in Non-Regenerative Multi-antenna Relay Selection Systems
}

\author{
S. Prakash and I. V. McLoughlin
}

May 16, 2012

\section{Introduction}

Co-operative relay technology appears to be a promising solution for future 4G (fourth generation) advanced wireless systems such as the 3GPP (Third Generation Partnership Program) LTE (Long Term Evolution) that aim to provide very high data rates and increased network coverage area [1-3]. Several information theoretic studies on MIMO-enabled (multi-input, multi-output) relaying, show improvements are possible in link reliability and spectral efficiency [4,5]. Infrastructure-based (fixed) multi-antenna relays have been found beneficial in providing diversity and capacity improvements for wireless terminals with limited number of antennas $[2,6]$. However, to achieve high performance, at least two receive antennas are generally recommended for future wireless system terminals [3]. In practical terms, source-directed relay selection, where a single best relay forwards the signal to the destination, overcomes multiple transmission needs, synchronisation issues, and excess bandwidth usage, in contrast to distributed/centralised relay selection schemes which can be time sensitive [7]. Furthermore, relay systems tend to have much lower implementation complexity [8] than full space-time coded alternatives. Fixed gain amplifyand-forward (AF) relay selection is particularly simple to implement compared to decode-and-forward, and has been well studied for single antenna devices [7, 9-12]. Non-selective beamforming for single antenna relay or destination devices is an alternative strategy [13], and Da Costa and Aissa [14] had explored a hybrid approach of multi-antenna source and destination communication with a single antenna relay. In general, published results reveal that partial selection performance does not improve much when more than two relay nodes are available for selection, and performance is limited by the gain of the second hop. This paper extends these analyses further to consider source, destination, and relay nodes equipped with multiple receive antennas, but using single transmit antennas. This is a source-directed arrangement with no direct path from source to destination.

In a source-selected arrangement like this, channel state information (CSI) measured at the relays is fed back on a frame-by-frame basis and used for relay selection at the transmitter (source). In general, the performance degrades as the fed back information becomes more outdated. In fact, any such system relying upon fed back information will experience a delay between obtaining the information and being able to act upon it. CSI becomes outdated due to phenomena such as Doppler changes, with the consequence of reduced selection accuracy. This effect has been explored in single antenna systems [15]. In general, the impact of delayed feedback on relay systems has been analysed [16], and recently the impact of outdated feedback information on relay selection has been considered $[10,17]$. Performance limits tend towards accurate selection with perfect knowledge at zero feedback delay, and the performance of a non-selective system (i.e. without CSI knowledge at the source) when feedback delays are greater than the channel fading time or coherence limit. 
This effect has also been explored in transmit antenna selection (i.e. non-relay) systems, where channel prediction has been shown to be a viable method of mitigating against degraded CSI [18].

This paper aims to (a) investigate performance improvement for multiple antennas over a single antenna for source-selected AF relays, (b) investigate the dual-hop AF relay system impact due to feedback delay, (c) to mitigate against the effect of degraded CSI by introducing a predictor able to exploit temporal channel correlation.

Closed-form solutions will be derived to characterise the system, which is also explored through simulation. Results will show that first hop quality improves with additional relays (or number of antennas), while second hop improvement comes about primarily as a result of increased receive diversity. The variable gain AF used in this system naturally exploits CSI, estimated at the relays (note that the performance of fixed and variable gain relay selection has been explored in [15]). Accurate channel estimates are assumed for the purpose of MRC combining and demodulation - justifiable since the effect of channel estimation error is often much less than that of using outdated CSI to make selection decisions. First hop bit error rate (BER) and probability density function (PDF) equations were derived in our previous work, on non-relayed channel prediction for point-to-point (PtP) linking [19] and thus not re-derived here.

\section{System model and assumptions}

Consider an AF relay network of physically identical nodes equipped with single transmitter and multiple receivers. Mobile source $M_{S}$ communicates to $N_{t}$ relay nodes $R_{1}, R_{2}, \ldots, R_{N_{t}}$ each with $N_{r r}$ receive antennas. A selected best relay $R_{l}, l$ : $1 \leq l \leq N_{t}$, chosen based on received SNR, forwards the amplified signal to mobile destination $M_{D}$ which has $N_{r d}$ receive antennas. No direct link exists between $M_{S}$ and $M_{D}$, and information is conveyed half duplex with transmission in two phases, $M_{S} \rightarrow R_{l} \rightarrow M_{D}$. The structure of the system is illustrated in Fig. 1. Block fading, with block length $L_{b}$ and symbol rate $T_{s}$, is assumed. Thus the channel matrix for the first hop at instant $k$ is $\mathbf{H}(k)=\left[h_{i j}(k)\right]_{N_{r r} \times N_{t}} 1 \leq j \leq N_{r r}, 1 \leq i \leq N_{t}$, with channels $h_{i j}$ characterised as spatially independent and identically distributed (i.i.d) complex Gaussian random variables with distribution $\mathcal{C N}(0,1)$. Channels are temporally correlated and change slowly over transmission blocks - the well known Jakes fading model is used, with $f_{d}$ as Doppler frequency. To obtain feedback information about the SNRs at the relays, $M_{S}$ can broadcast pilot channel information as well as data using pilot symbol aided modulation (PSAM). The estimated channel matrix between $M_{S}$ and relays is then $\widetilde{\mathbf{H}}(k)=\left[\tilde{h}_{i j}(k)\right]_{N_{r r} \times N_{t}}$ where $\tilde{h}_{i j}(k)$ is the channel estimate distribution $\mathcal{C} \mathcal{N}\left(0,1+\sigma_{v}^{2}\right)$, (assuming estimation and channel noise to be independent) with $\sigma_{v}^{2}=N_{0} / E_{p}$ as variance of estimation error, $E_{p}$ being the power of the pilot symbol and $N_{0}$ the AWGN (additive white Gaussian noise) variance, assumed to be the same for each transmit-receive chain. To compensate for known feedback delay $\mathcal{D}$, each relay $R_{i}$ independently predicts combined branch power for a future transmission slot using a Wiener-Hopf filter based on noisy estimated CSI at its receiver. This is conveyed to the source through a feedback link.

The predicted SNR at the $i^{\text {th }}$ relay is then given as;

$$
\hat{\gamma}_{i}(k+\mathcal{D})=\frac{\mathcal{E}_{1}}{N_{0}} \sum_{j=1}^{N_{r r}}\left|\hat{h}_{i j}(k+\mathcal{D})\right|^{2}
$$

where $\mathcal{E}_{1}$ is the power of the transmitted signal at $M_{S}$ and $\hat{h}_{i j}(k+\mathcal{D})=\mathbf{w}_{o p t}^{H} \widetilde{\mathbf{h}}_{i j}$ is the $\mathcal{D}$ block ahead predicted channel coefficient. $\widetilde{\mathbf{h}}_{i j}$ is the complex vector of estimated 
fading amplitudes corresponding to prediction length $L$.

$$
\widetilde{\mathbf{h}}_{i j}=\left[\tilde{h_{i j}}(k), \tilde{h_{i j}}(k-1), \ldots, \tilde{h_{i j}}(k-(L-1)]^{T}\right.
$$

and $\mathbf{w}_{\text {opt }}$ is the Wiener optimal complex coefficient vector $\mathbf{w}_{\text {opt }}=[\mathbf{R}]^{-1} \mathbf{r}$ where $[\mathbf{R}]_{\varphi \vartheta}=J_{0}\left(2 \pi f_{d}|\varphi-\vartheta| L_{b} T_{s}\right)+\sigma_{v}^{2} \delta(\varphi-\vartheta)$, and $\mathbf{r}_{\varphi}=J_{0}\left(2 \pi f_{d}|\mathcal{D}+\varphi-1| L_{b} T_{s}\right) ; \varphi, \vartheta=$ $1,2, \ldots, L . J_{0}($.$) is the zeroth order Bessel function of the first kind. (.) { }^{H}$ denotes the Hermetian transpose. Based upon computed $\hat{\gamma}_{i}$ fed back to $M_{S}$, a single best relay offering best predicted instantaneous SNR, with index $l$, is selected for the current interval: $\hat{\gamma}_{l}(k+\mathcal{D})=\max _{1 \leq i \leq N_{t}} \hat{\gamma}_{i}(k+\mathcal{D})$. This relay combines the received signal using MRC. The same initial PSAM estimates are used for channel smoothing, but will yield a cleaner channel estimate, so for MRC combining at the selected relay we assume $\tilde{h}_{l j}(k)=h_{l j}(k), 1 \leq j \leq N_{r r}$ denoted as $\left[\mathbf{h}_{M_{S} R_{l}}\right]_{N_{r r} \times 1}$. This will be the first hop path $M_{S} \rightarrow R_{l}$, with instantaneous MRC weight vector;

$$
\mathbf{w}_{M_{S} R_{l}}=\frac{\mathbf{h}_{M_{S} R_{l}}^{H}}{\sqrt{\mathbf{h}_{M_{S} R_{l}}^{H} \mathbf{h}_{M_{S} R_{l}}}} .
$$

The signal is then boosted by variable gain $G$ and conveyed to the destination over the second hop $R_{l} \rightarrow M_{D}$ channel $\left[\mathbf{h}_{R_{l} D}\right]_{N_{r d} \times 1}$, which is $\mathcal{C N}\left(0, \mathbf{I}_{N_{r d}}\right)$ distributed, also assumed correctly estimated for signal demodulation at $M_{D}$ (I is an identity matrix) and

$$
\mathbf{w}_{R_{l} M_{D}}=\frac{\mathbf{h}_{R_{l} M_{D}}^{H}}{\sqrt{\mathbf{h}_{R_{l} M_{D}}^{H} \mathbf{h}_{R_{l} M_{D}}}}
$$

gives the MRC weight vector. The final combined output SNR at the $M_{D}$ is then

$$
\begin{array}{r}
y_{R_{l} M_{D}}=G \mathbf{w}_{R_{l} M_{D}} \mathbf{h}_{R_{l} M_{D}}\left(\mathbf{w}_{M_{S} R_{l}} \mathbf{h}_{M_{S} R_{l}} s+\mathbf{w}_{M_{S} R_{l}} \mathbf{n}_{R_{l}}\right) \\
+\mathbf{w}_{R_{l} M_{D}} \mathbf{n}_{M_{D}}
\end{array}
$$

where $s$ is the transmitted symbol, $\mathbf{n}_{R_{l}}$ and $\mathbf{n}_{M_{D}}$ are noise vectors at relay and destination, characterised as $\mathcal{C N}\left(0, N_{0} \mathbf{I}_{N_{r r}}\right)$ and $\mathcal{C N}\left(0, N_{0} \mathbf{I}_{N_{r d}}\right)$. After simplification of (5) [20], the equivalent SNR at $M_{D}$ can then be calculated as $\gamma_{e q}=\frac{\gamma_{1} \gamma_{2}}{\gamma_{1}+\gamma_{2}+1}$ with

$$
G^{2}=\frac{\mathcal{E}_{2}}{\mathcal{E}_{1}\left(\mathbf{h}_{M_{S} R_{l}}^{H} \mathbf{h}_{M_{S} R_{l}}\right)+N_{0}}
$$

and where

$$
\gamma_{1}=\frac{\mathcal{E}_{1} \mathbf{h}_{M_{S} R_{l}}^{H} \mathbf{h}_{M_{S} R_{l}}}{N_{0}}
$$

and

$$
\gamma_{2}=\frac{\mathcal{E}_{2} \mathbf{h}_{R_{l} M_{D}}^{H} \mathbf{h}_{R_{l} M_{D}}}{N_{0}}
$$

are the instantaneous SNRs of each hop respectively. Average receive SNR per symbol per path for first and second hop is $\bar{\gamma}_{1}=\mathcal{E}_{1} / N_{0}$ and $\bar{\gamma}_{2}=\mathcal{E}_{2} / N_{0}$ respectively, with $\mathcal{E}_{2}$ being the power of the transmitted signal at $R_{l}$. In the next section we will formulate fading statistics for the two hops in order to derive the important outage probability and SER metrics.

\section{Performance metrics}

The outage probability $P_{\text {out }}$ is defined as the probability that instantaneous SNR is less than a given threshold $\gamma_{T}$ [20], a useful measure of real-world system performance. 
In a variable $\mathrm{AF}$ relay system this is generalised as:

$$
\begin{aligned}
P_{\text {out }}\left(\gamma_{T}\right) & =P_{\text {out }}\left(\gamma_{e q}<\gamma_{T}\right) \\
& =\int_{0}^{\infty} \operatorname{Pr}\left[\frac{\gamma_{1} \gamma_{2}}{\gamma_{1}+\gamma_{2}+1}<\gamma_{T} \mid \gamma_{2}\right] f_{\gamma_{2}}\left(\gamma_{2}\right) d \gamma_{2}
\end{aligned}
$$

The above can also be rearranged into a complementary form which will be more convenient for later manipulation:

$$
P_{\text {out }}\left(\gamma_{T}\right)=1-\int_{\gamma_{T}}^{\infty}\left[1-F_{\gamma_{1}}\left(\frac{\gamma_{T}\left(\gamma_{2}+1\right)}{\gamma_{2}-\gamma_{T}}\right)\right] f_{\gamma_{2}}\left(\gamma_{2}\right) d \gamma_{2}
$$

where $F_{\gamma_{1}}(\cdot)$ is the CDF (cumulative distribution function) of $\gamma_{1}$. We now need to obtain an expression for $F_{\gamma_{1}}(\cdot)$ by first deriving its PDF, $f_{\gamma_{1}}(\gamma)$. Let $f_{\hat{\gamma}_{i}}(\hat{\gamma})$ be the PDF of the i.i.d random variables corresponding to the predicted SNRs of $S \rightarrow R_{i=1 \ldots, N_{t}}$ links. Each $f_{\gamma_{i}}(\hat{\gamma})$ is a gamma PDF [19] $\hat{\gamma}_{i} \sim \mathcal{G}\left(N_{r r}, \overline{\hat{\gamma}}\right)$ with $\overline{\hat{\gamma}}=\bar{\gamma}_{1} \mathbf{r}^{\mathbf{H}} \mathbf{R}^{-\mathbf{1}} \mathbf{r}$. Arranging the random variables as $\hat{\gamma}_{(1)} \leq \hat{\gamma}_{(2)} \leq \cdots \leq \hat{\gamma}_{\left(N_{t}\right)}$, the relay corresponding to the $N_{t}^{\text {th }}$ order statistic will be selected. Since $\hat{\gamma}_{i}$ are assumed to be i.i.d, then according to order statistics the PDF of the predicted maximum SNR or $\hat{\gamma}_{l}$, is $^{1} f_{\hat{\gamma}_{l}}(\hat{\gamma})=N_{t} f_{\hat{\gamma}}(\hat{\gamma})\left[F_{\hat{\gamma}}(\hat{\gamma})\right]^{N_{t}-1}[19] . F_{\hat{\gamma}}(\hat{\gamma})$ is the CDF of $\hat{\gamma}$ and can easily be shown to be:

$$
F_{\hat{\gamma}}(\hat{\gamma})=1-\exp \left(-\hat{\gamma} / \bar{\gamma}_{1}\right) \sum_{m=0}^{N_{r r}-1}(1 / m !)\left(\hat{\gamma} / \bar{\gamma}_{1}\right)^{m}
$$

The PDF of $\gamma_{1}$ is given as:

$$
f_{\gamma_{1}}(\gamma)=\int_{0}^{\infty} f_{\gamma \mid \hat{\gamma}}(\gamma \mid \hat{\gamma}) f_{\hat{\gamma}_{l}}(\hat{\gamma}) d \hat{\gamma}
$$

where the corresponding true SNR $\gamma$ has PDF $\gamma \sim \mathcal{G}\left(N_{r r}, \bar{\gamma}_{1}\right)$ and the conditional density $f_{\gamma \mid \hat{\gamma}}(\gamma \mid \hat{\gamma})=f_{\gamma, \hat{\gamma}}(\gamma, \hat{\gamma}) / f_{\hat{\gamma}}(\hat{\gamma})$ where $f_{\gamma \mid \hat{\gamma}}(\gamma, \hat{\gamma})$ is a bi-variate gamma PDF [19] $\mathcal{G}_{B}\left(N_{r r}, \bar{\gamma}_{1}, \overline{\hat{\gamma}}, \rho_{p}\right)$ with $\rho_{p}=\mathbf{r}^{\mathbf{H}} \mathbf{R}^{-1} \mathbf{r}$. Using multinomial and binomial expansions along with [21, eqn.(6.643.4)], a closed form solution of $f_{\gamma_{1}}(\gamma)$ can be obtained in terms of Laguerre polynomials [21]. $F_{\gamma_{1}}(z)$ is then found as $F_{\gamma_{1}}(z)=\int_{0}^{z} f_{\gamma_{1}}(\gamma) d \gamma$ using [21, eqn.(8.350)]. After rearrangement, it is then advantageous to cast $F_{\gamma_{1}}(z)$ in complementary CDF form to match (10) as:

$$
\begin{aligned}
& F_{\gamma_{1}}(z)=1-\frac{N_{t} !}{\left(N_{r r}-1\right) !} \sum_{i=0}^{N_{t}-1} \frac{(-1)^{i} \exp \left(-\frac{(i+1)\left(z / \bar{\gamma}_{1}\right)}{\left[\left(i\left(1-\rho_{p}\right)+1\right)\right]}\right)}{i !\left(N_{t}-1-i\right) !} \\
& \cdot \sum_{j=0}^{i\left(N_{r r}-1\right)} \eta_{N_{r r}}(i, j)\left(N_{r r}+j-1\right) ! \sum_{k=0}^{j}\left(\begin{array}{l}
j \\
k
\end{array}\right) \frac{\rho_{p}^{k}\left(1-\rho_{p}\right)^{j-k}}{\left[\left(i\left(1-\rho_{p}\right)+1\right)\right]^{j}} \\
& \cdot \sum_{l=0}^{k+N_{r r}-1} \frac{\left(z / \bar{\gamma}_{1}\right)^{l}}{l !(i+1)^{k+N_{r r}-l}\left[i\left(1-\rho_{p}\right)+1\right]^{l}}
\end{aligned}
$$

where $\eta_{N_{r r}}(i, j)\left[21\right.$, eqn.(0.314)] is the coefficient of $\lambda^{j}, j=0,1, \ldots i\left(N_{r r}-1\right)$, in the expansion of $\left(\sum_{j=0}^{N_{r r}-1} \lambda^{j} / j !\right)^{i}$. The PDF of the second hop $R_{l} \rightarrow D, f_{\gamma_{2}}(\gamma)$, is a well known gamma PDF, which is $\mathcal{G}\left(N_{r d}, \bar{\gamma}_{2}\right)$ :

$$
f_{\gamma_{2}}\left(\gamma_{2}\right)=\frac{\gamma_{2}^{N_{r d}-1}}{\bar{\gamma}_{2}{ }^{N_{r d}}\left(N_{r d}-1\right) !} \exp \left(-\frac{\gamma_{2}}{\bar{\gamma}_{2}}\right)
$$

\footnotetext{
${ }^{1}$ Index $i$ is dropped since they have identical distributions, and time indices are omitted assuming a stationary random process.
} 
Changing variables in (10) enables changing limits from 0 to $\infty$. Using $f_{\gamma_{2}}\left(\gamma_{2}\right)$ and (13) in (10), we expand out inner binomial terms containing $\gamma_{T}$. Simplifying, and evaluating the integral using [21, eqn.(3.471.9)], an exact expression for $P_{\text {out }}$ becomes a function of $\mathbf{K}_{v}($.$) , the v^{\text {th }}$ order modified Bessel function of the second kind [21].

$$
\begin{aligned}
& P_{\text {out }}\left(\gamma_{T}\right)=1-\frac{2 N_{t} !}{\bar{\gamma}_{2}^{N_{r d}}\left(N_{r r}-1\right) !} \sum_{i=0}^{N_{t}-1} \frac{(-1)^{i}}{i !\left(N_{t}-1-i\right) !} \\
& \cdot \exp \left(-\frac{(i+1) \gamma_{t h}}{\left[\left(i\left(1-\rho_{p}\right)+1\right) \bar{\gamma}_{1}\right]}-\frac{\gamma_{t h}}{\bar{\gamma}_{2}}\right) \\
& \sum_{j=0}^{i\left(N_{r r}-1\right)} \eta_{N_{r r}}(i, j)\left(N_{r r}+j-1\right) ! \sum_{k=0}^{j}\left(\begin{array}{l}
j \\
k
\end{array}\right) \frac{\rho_{p}^{k}\left(1-\rho_{p}\right)^{j-k}}{\left[\left(i\left(1-\rho_{p}\right)+1\right)\right]^{j}} \\
& \cdot \sum_{l=0}^{k+N_{r r}-1} \frac{\left[\gamma_{T} /\left(i\left(1-\rho_{p}\right)+1\right) \bar{\gamma}_{1}\right]^{l}}{l !(i+1)^{k+N_{r r}-l}} \sum_{p=0}^{l} \frac{l !\left(\gamma_{T}+1\right)^{l-p}}{(l-p) ! p !} \\
& \cdot \sum_{q=0}^{N_{r d}-1} \frac{\gamma_{T}^{N_{r d}-q}}{\left(N_{r d}-1-q\right) ! q !}\left[\frac{\bar{\gamma}_{2}(i+1) \gamma_{T}\left(1+\gamma_{T}\right)}{\left(i\left(1-\rho_{p}\right)+1\right) \bar{\gamma}_{1}}\right]^{\frac{(q+p-l+1)}{2}} \\
& \cdot \mathbf{K}_{(q+p-l+1)}\left(2 \sqrt{\frac{(i+1) \gamma_{T}\left(1+\gamma_{T}\right)}{\left(i\left(1-\rho_{p}\right)+1\right) \bar{\gamma}_{1} \bar{\gamma}_{2}}}\right)
\end{aligned}
$$

Next, the average SER for coherent demodulation in a slow fading channel is found utilizing the $\mathrm{CDF} F_{\gamma_{e q}}(\cdot)$ (or $\left.P_{\text {out }}(\cdot)\right)[9]$ :

$$
\bar{P}_{s}=\frac{\alpha}{\sqrt{(2 \pi)}} \int_{0}^{\infty} F_{\gamma_{e q}}\left(\frac{x^{2}}{\beta}\right) \exp \left(\frac{-x^{2}}{2}\right) d x
$$

where $\alpha, \beta$ are chosen to define specific modulation formats [9]. By approximating $\gamma_{T} \approx \gamma_{T}+1$ in (15) setting $\gamma_{T}=x^{2} / \beta$, the above integral can be solved using [21, eqn.(6.621.3)] to obtain a closed form solution for $\bar{P}_{s}$;

$$
\begin{gathered}
\bar{P}_{s} \approx \frac{\alpha}{\sqrt{2 \pi}}\left[\frac{\sqrt{2 \pi}}{2}-\frac{\sqrt{\pi \beta}\left(N_{r d}-1\right) ! N_{t} !}{\bar{\gamma}_{2}\left(N_{r r}-1\right) !} \sum_{i=0}^{N_{t}-1} \frac{(-1)^{i}}{i !\left(N_{t}-1-i\right) !}\right. \\
\sum_{j=0}^{i\left(N_{r r}-1\right)} \eta_{N_{r r}}(i, j)\left(N_{r r}+j-1\right) ! \sum_{k=0}^{j}\left(\begin{array}{l}
j \\
k
\end{array}\right) \frac{\rho_{p}^{k}\left(1-\rho_{p}\right)^{j-k}}{\left[\left(i\left(1-\rho_{p}\right)+1\right)\right]^{j}} \\
\sum_{q=0}^{N_{r d}-1} \frac{\Gamma\left[N_{r d}-q-p+2 l-0.5\right]}{\left.\sum_{l=0}^{k+N_{r r}-1} \frac{\left(\Gamma\left[l+N_{r d}+1\right]\right)^{-1}}{l !(i+1)^{k+N_{r r}-l}\left[i\left(1-\rho_{p}\right)+1\right]^{l} \bar{\gamma}_{1}^{l}} \sum_{p=0}^{l} \frac{4(i+1)}{\left(i\left(1-\rho_{p}\right)+1\right) \bar{\gamma}_{1} \bar{\gamma}_{2}}\right]^{(q+p-l+1) ! p !}} \\
\frac{\Gamma\left[N_{r d}+q+p+1.5\right]}{\left[\frac{i+1}{\left(i\left(1-\rho_{p}\right)+1\right) \bar{\gamma}_{1}}+\frac{1}{\bar{\gamma}_{2}}+\frac{\beta}{2}+2 \sqrt{\frac{i+1}{\left(i\left(1-\rho_{p}\right)+1\right) \bar{\gamma}_{1} \bar{\gamma}_{2}}}\right]^{N_{r d}+q+p+1.5}} \\
\left.{ }_{2} \mathbf{F}_{1}\left(N_{r d}+q+p+1.5, q+p-l+1.5 ; l+N_{r d}+1 ; \xi\right)\right]
\end{gathered}
$$

where $\boldsymbol{\Gamma}[\cdot]$ denotes the Gamma Function, and ${ }_{2} \mathbf{F}_{1}(\cdot, \cdot, \cdot ; \cdot)$ is the Gauss hypergeo- 
metric function [21] with

$$
\xi=\frac{\left[\frac{i+1}{\left(i\left(1-\rho_{p}\right)+1\right) \bar{\gamma}_{1}}+\frac{1}{\bar{\gamma}_{2}}+\frac{\beta}{2}-2 \sqrt{\frac{i+1}{\left(i\left(1-\rho_{p}\right)+1\right) \bar{\gamma}_{1} \bar{\gamma}_{2}}}\right]}{\left[\frac{i+1}{\left(i\left(1-\rho_{p}\right)+1\right) \bar{\gamma}_{1}}+\frac{1}{\bar{\gamma}_{2}}+\frac{\beta}{2}+2 \sqrt{\frac{i+1}{\left(i\left(1-\rho_{p}\right)+1\right) \bar{\gamma}_{1} \bar{\gamma}_{2}}}\right]} .
$$

This approximation implicit in this closed form solution holds good in the moderate to high SNR regions.

\section{Numerical and simulation results}

BER performance is evaluated and verified through simulation. $P_{\text {out }}\left(\gamma_{T}\right)$ in $(15)$ is directly verified by this (since BER is derived using it), but not plotted here due to space constraints. In Fig. 2, BER for BPSK $(\alpha=1, \beta=2)$ in different relay configurations, denoted as $\left(N_{t}, N_{r r}, N_{r d}\right)$ are shown against $\bar{\gamma}_{1}$ for correlations ${ }^{2}$ of $\rho_{p}=1,0.9,0.7$, where $\rho_{p}=1$ implies no delay or near perfect prediction. We set $\bar{\gamma}_{1}=5 \bar{\gamma}_{2}$ to simulate a disparity between first and second hop channel gains. This is realistic since it would be rare that a deployed system experiences identical gains over both hops.

In general, it is known that using multiple antennas and relays can lead to large performance improvements, however this is not necessarily true in every case since performance can be limited by channel strength over one particular hop (as discussed below). As seen for any given $\bar{\gamma}_{1}$ in the selection plots, BER degrades, as $\rho_{p}$ decreases. For large delays, correlation tends to zero and diversity gains from relay switching drop to zero, just like a single relay system. For example the $(3,1,1)$ and $(2,2,2)$ results tend towards $(1,1,1)$ and $(1,2,2)$ respectively, as $\rho_{p} \rightarrow 0$, meaning that any gain obtained through having multiple relays is lost because the system no longer has the capability of selecting a best relay. The $(2,2,2)$ scheme has a diversity order ${ }^{3}$ of 2 for large SNRs, determined by the minimum diversity order of the first and the second hop (the result follows from Lemma 3 in [5]). In other words, if the first hop channel strength is greater than the second, then increasing $N_{r d}$ will be beneficial. By contrast, if second hop diversity gain is strong, then increasing $N_{r r}$ or $N_{t}$ will improve performance. We can see that the $(1,3,1)$ system is better than the delaylimited $(3,1,1)$ scheme at low SNRs, but both converge at large SNRs. It means that in an SNR-constrained system, it may be better to have just one relay with multiple antennas, rather than multiple relays with single antennas. Also, note one practical consideration: the feedback bandwidth of the $(3,1,1)$ scheme is 3 times that of $(1,3,1)$ and 1.5 times more than either $(2,2,2)$ or $(2,2,3)$. Similarly, the $(2,2,3)$ result tends toward $(1,2,3)$ at a faster rate, since its diversity order now reduces from 3 for no delay to 2 when delayed. This is expected because again the loss of switching accuracy negates the benefit of having multiple relays.

Fig. 3 highlights this loss due to delay more clearly, where BER for various setups with $\bar{\gamma}_{1}=8,15 d B$ is plotted. Normalised delay $f_{d} \tau$, with $\tau=\mathcal{D} L_{b} T_{s}$, ranges from a very low $10^{-2}$ to a large value of 3 . The plot shows the effect of each setup. Note for the non-predictive case, it can be shown that eqn. (15) and (17) hold true, with $\rho_{p}=J_{0}^{2}\left(2 \pi f_{d} \tau\right)$. Firstly, the predicted case is evidently superior than the conventional case, tolerating larger values of $f_{d} \tau$. For very large $f_{d} \tau$, both predicted and non-predictive schemes converge to their corresponding configurations as if just

\footnotetext{
${ }^{2}$ Note $\rho_{p}$ is a function of several variables, finding an appropriate set for any given $\mathcal{D}$ is a general optimisation problem [19].

${ }^{3}$ The first hop is analogous to PtP uncoded best-antenna TAS/MRC. With delay free switching of $T$ transmit antennas $/ R$ receive chains, it is known that diversity order $G_{d}=T R$. In the delayed case, it can be proven that $G_{d}=R$.
} 
one relay is used. For example, $(2,1,1)$ and $(7,1,1)$ both tend towards $(1,1,1)$ under high delay conditions while $(2,2,2),(3,2,2)$ and $(4,2,2)$ tend towards $(1,2,2)$. We can thus see that prediction can be very beneficial in preserving spatial diversity gains realised through relay selection. As seen at lower $f_{d} \tau$, not much improvement is seen going from $(2,1,1)$ to $(7,1,1)$ with most gains coming from just two relays (a previously published result, mentioned in Section 1), however we can see something additional here: the former is more delay sensitive than the latter. Thus an important result is that adding relays improves delay tolerance in both predictive and nonpredictive cases. Interestingly, increasing $N_{r r}$ is also effective in lowering BER and tolerating delay (see $(2,3,2)$ and $(2,4,2)$ at high SNR where little degradation is seen). Note that, at low $f_{d} \tau$ or no delay, and $\bar{\gamma}_{1}$, while increasing $N_{t}, N_{r r}$ lowered BER in general, saturation occurred quickly at high SNRs: performance can then be improved only through increasing $N_{r d}$ or $G$ (to strengthen the second hop). At high $f_{d} \tau$, increasing $N_{t}, N_{r r}$ counters degradation due to delay - increasing $N_{r d}$ improves BER, but makes the system more delay sensitive. Fig. 2 showed this for $(2,2,2)$ and $(2,2,3)$. Also, fig. 3 shows that BER does not improve when $N_{r d}$ exceeds the first hop diversity order: in delay limited cases, saturation occurs earlier. However, sensitivity to increasing resources, such as $N_{t}$ and $N_{r r}$ or $N_{r d}$, depends on the ratio $\bar{\gamma}_{1}: \bar{\gamma}_{2}$, i.e. the relative hop strengths. A higher ratio decreases sensitivity to $N_{t}, N_{r r}$ but increases sensitivity to $N_{r r}$. In an implementation of a dual-hop AF relaying system such as the one described, careful balancing of resources including antennas, number and placement of relays, along with transmit power adjustment, would be necessary to maximise performance. The closed-form solutions derived here aid in such system balancing and performance trade-off optimisation.

\section{Conclusion}

Closed form BER and outage probability expressions were derived and explored for a dual-hop multi-antenna relay selection system experiencing various degrees of CSI feedback delay. Results have shown that while adding a small number of antennas or relays into the system can improve performance, a careful distribution of antenna resources is needed to realise substantial gains over a single antenna relay system. In terms of switching delay, increasing the number of relays or relay receive antennas can overcome some of the loss due to moderately outdated switching information. However, prediction was shown to be particularly beneficial in preserving the spatial diversity gains that are promised by the provision of multiple relays when performing relay selection under delay-limited feedback conditions (or across quickly-changing channels).

\section{References}

[1] J. N. Laneman and G. W. Wornell, "Energy-efficient antenna sharing and relaying for wireless networks," in Proc. IEEE WCNC 2000, Oct. 2000, pp. $7-12$ vol.1.

[2] R. Pabst et al., "Relay-based deployment concepts for wireless and mobile broadband radio," IEEE Commun. Mag., vol. 42, no. 9, pp. 80 - 89, Sept. 2004.

[3] S. Parkvall and D. Astely, "The Evolution of LTE towards IMT-Advanced," Journal of Comm., vol. 4, no. 3, p. 146, 2009.

[4] H. Bolcskei, R. U. Nabar, O. Oyman, and A. J. Paulraj, "Capacity scaling laws in MIMO relay networks," IEEE Trans. Wireless Commun., vol. 5, no. 6, pp. $1433-1444$, Jun. 2006. 
[5] M. Yuksel and E. Erkip, "Multiple-antenna cooperative wireless systems: A diversity;multiplexing tradeoff perspective," IEEE Trans. Inf. Theory, vol. 53, no. 10, pp. $3371-3393$, Oct. 2007.

[6] A. Adinoyi and H. Yanikomeroglu, "Cooperative relaying in multi-antenna fixed relay networks," IEEE Trans. Wireless Commun., vol. 6, no. 2, pp. $533-544$, Feb. 2007.

[7] I. Krikidis, J. Thompson, S. McLaughlin, and N. Goertz, "Amplify-and-forward with partial relay selection," IEEE Commun. Lett., vol. 12, no. 4, pp. 235 -237, Apr. 2008.

[8] A. Bletsas, A. Khisti, D. P. Reed, and A. Lippman, "A simple cooperative diversity method based on network path selection," IEEE J. Sel. Areas Commun., vol. 24, no. 3, pp. 659 - 672, Mar. 2006.

[9] Y. Zhao, R. Adve, and T. J. Lim, "Symbol error rate of selection amplify-andforward relay systems," IEEE Commun. Lett., vol. 10, no. 11, pp. $757-759$, Nov. 2006.

[10] H. A. Suraweera, M. Soysa, C. Tellambura, and H. Garg, "Performance analysis of partial relay selection with feedback delay," IEEE Signal Process. Lett., vol. 17 , no. 6 , pp. $531-534$, Jun. 2010.

[11] D. B. da Costa and S. Aissa, "Performance analysis of relay selection techniques with clustered fixed-gain relays," IEEE Signal Process. Lett., vol. 17, no. 2, pp. $201-204$, Feb. 2010.

[12] S. Prakash and I. McLoughlin, "Performance of Dual-Hop Multi-Antenna Systems with Fixed Gain Amplify-and-Forward Relay Selection," in IEEE Transactions on Wireless Communications, vol. 10, no. 6, June 2011, pp. 1709 - 1714.

[13] H. Min, S. Lee, K. Kwak, and D. Hong, "Effect of multiple antennas at the source on outage probability for amplify-and-forward relaying systems," IEEE Trans. Wireless Commun., vol. 8, no. 2, pp. 633 -637, Feb. 2009.

[14] D. da Costa and S. Aissa, "Cooperative dual-hop relaying systems with beamforming over Nakagami-m fading channels," IEEE Trans. Wireless Commun., vol. 8, no. 8, pp. 3950 -3954, Aug. 2009.

[15] A. Soysa, H. Suraweera, C. Tellambura, and H. Garg, "Partial and opportunistic relay selection with outdated channel estimates," IEEE Trans. Comms., vol. 60, to appear 2012.

[16] H. Suraweera, T. Tsiftsis, G. Karagiannidis, and M. Faulkner, "Effect of feedback delay on downlink amplify-and-forward relaying with beamforming," in Proc. IEEE GLOBECOM 2009, Dec. 2009, pp. 1-6.

[17] D. Michalopoulos, H. Suraweera, G. Karagiannidis, and S. R., "Amplify-andforward relay selection with outdated channel state information," Proc. IEEE GLOBECOM, pp. 1-6, 2010.

[18] S. Prakash and I. McLoughlin, "Effects of channel prediction for transmit antenna selection with maximal-ratio combining in rayleigh fading," in IEEE Transactions on Vehicular Technology, vol. 60, no. 6, July 2011, pp. $2555-$ 2568.

[19] _ - "Predictive Transmit Antenna Selection with Maximal Ratio Combining," in Proc. IEEE GLOBECOM 2009, Nov.30-Dec. 4 2009, pp. 1-6.

[20] M. O. Hasna and M.-S. Alouini, "A performance study of dual-hop transmissions with fixed gain relays," IEEE Trans. Wireless Commun., vol. 3, no. 6, pp. 1963 - 1968, Nov. 2004.

[21] I. S. Gradshteyn and I. M. Ryzhik, Table of Integrals, Series, and Products, 6th ed. New York: Academic, 2000, 2000. 


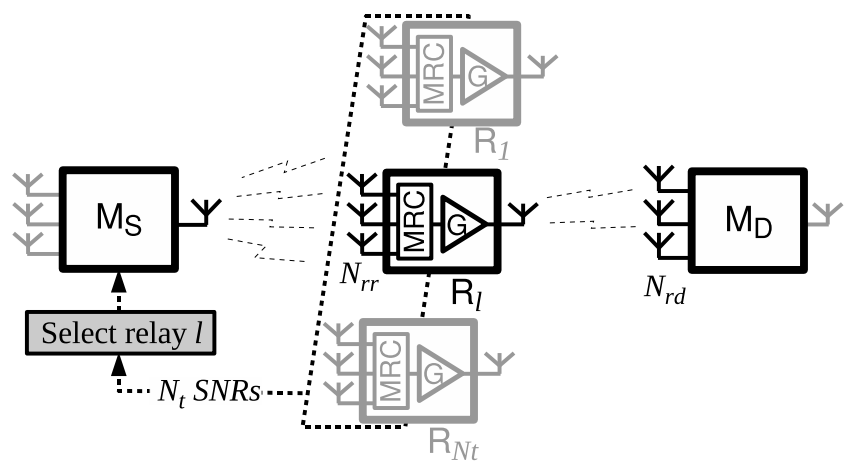

Figure 1: System arrangement showing $N_{t}$ identical but scattered relays each equipped with $N_{r r}$ receive antennas. A relay selected by source $M_{S}$ based upon first hop SNR is chosen to transmit amplified message data to destination $M_{D}$ which also employs MRC over $N_{r d}$ antennas. Setting $r r=r d$ allows a system constructed entirely from $N_{t}+2$ identical wireless devices.

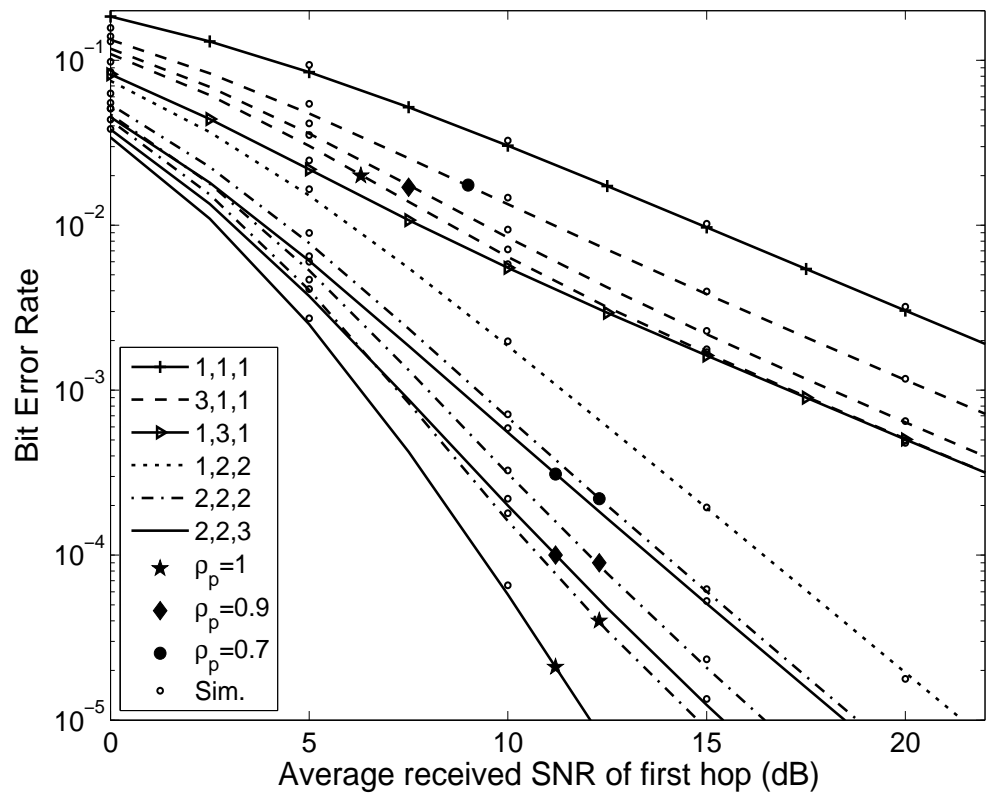

Figure 2: BER plot for BPSK under predictive selective relaying, for first hop temporal correlations of $\rho_{p}=1,0.9,0.7$, comparing single antenna and multi-antenna relay switching. 

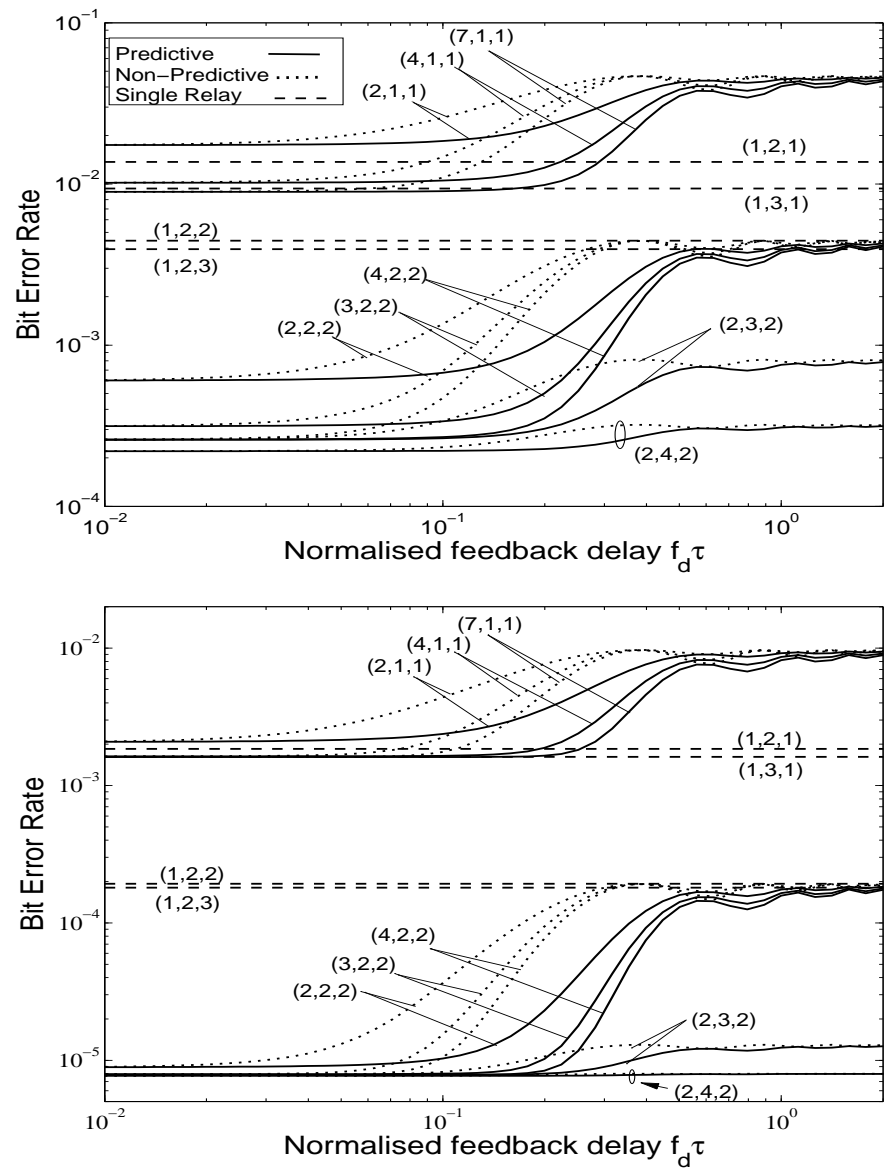

Figure 3: BER degradation due to feedback delay with and without prediction at $\bar{\gamma}_{1}=8 \mathrm{~dB}$ (top) and $\bar{\gamma}_{1}=15 d B$ (bottom) for different relay schemes with $\bar{\gamma}_{2}=5 \bar{\gamma}_{1}$. 